\title{
Ausschreibung des Heinz Maier-Leibnitz-Preises zur Förderung des wissenschaftlichen Nachwuchses
}

Der Bundesminister für Bildung und Wissenschaft stiftet im Rahmen seiner Förderung des wissenschaftlichen Nachwuchses den Heinz Maier-Leibnitz-Preis für wissenschaftlich hervorragende Originalveröffentlichungen. In Zusammenarbeit mit der Deutschen Forschungsgemeinschaft (DFG) sind für das Jahr 1992 die Gebiete

- Physik und Chemie des tiefen Erdinnern

- Wissenschafts- und Bildungsgeschichte

- Keramische Werkstoffe ausgewählt worden.

Als Summe können bis zu 12000 DM je Preisträger vergeben werden. Insgesamt stehen bis zu 185000 DM zur Verfügung.

Die Preise werden für Arbeiten verliehen, die in den letzten drei Jahren vor dem Schlußtermin dieser Ausschreibung aufgrund eines wissenschaftlichen Auswahlverfahrens in einer Zeitschrift, in einer anderen Sammelpublikation oder einer wissenschaftlichen Reihe erschienen bzw. zur Veröffentlichung angenommen sind.

Die Preise werden in der Regel an einzelne Autoren verliehen. Diese sollen bei der Annahme ihrer Arbeit zur Publikation das 33. Lebensjahr noch nicht überschritten haben. Die Vorgeschlagenen müssen deutsche Staatsangehörige sein oder ihren ständigen Wohnsitz und Arbeitsort in der Bundesrepublik Deutschland haben. Wird eine Arbeit mehrerer Autoren vorgelegt, so muß der überragende Beitrag der/des Vorgeschlagenen belegt werden.

Selbstbewerbung ist nicht möglich. Vorschlagsberechtigt sind Hochschullehrer und Wissenschaftler in entsprechender Stellung an Institutionen außerhalb der Hochschulen.

Vorschläge mit Sonderdrucken oder lesbaren Kopien (möglichst sechsfach), einer knappen Begründung des Vorschlags sowie gegebenenfalls ergänzende Angaben zum wissenschaftlichen Werdegang (kurzer Lebenslauf, evtl. Publikationsverzeichnis) werden bis spätestens

28. Februar 1992

an den Bundesminister für Bildung und Wissenschaft, Heinemannstraße 2, 5300 Bonn 2

erbeten.

Die Preise werden nach Beurteilung durch eine aus Fachgelehrten der einzelnen Gebiete gebildeten Jury im Sommer 1992 vergeben. Die Verleihung erfolgt im Rahmen einer öffentlichen Veranstaltung. 